\title{
Relationship between Time Length of E-learning Environment Using and Physical Health of Elementary and Middle School Students
}

\author{
Jing Gao, ${ }^{1}$ Jing Liu, ${ }^{2}$ Hong Feng, ${ }^{1}$ Zhixin Zhang $^{1}$ \\ 1. Capital Normal University, Beijing, China \\ 2. Beijing Open University, Beijing, China
}

\begin{abstract}
A total of 783 elementary and middle school students in Beijing city and Shandong province were selected for a one-year follow-up investigation, and the impact of time length of using E-learning environment each week on physical health was explored from four aspects as eyesight, respiratory rate, body mass index, sit and reach. Through Covariance Analysis, we found that time length of using E-learning environment each week by elementary and middle school students had a significant impact on their eyesight, but not on the respiratory rate, body mass index, sit and reach. Meanwhile, the comparative results of elementary and middle school students between different genders and learning stages that affected by the time length of E-learning were basically consistent with the findings above. Slight difference was found that the E-learning environment produced significant impact on both left and right eyesight of elementary girls, but on the left eyesight of middleschool boys, and significant effect on the middle-school body mass index. Based on this, we proposed that the time length of E-learning each day for elementary and middle school students should be controlled less than 1.5 hours.
\end{abstract}

Best Evid Chin Edu 2019; 3(1):277-286.

Doi: 10.15354/bece.19.ar1062

Keywords: E-Learning Environment; Eyesight; Respiratory Rate; Body Mass Index; Sit and Reach

Correspondence to: Jing Liu, Assistant Research Fellow, OUCHN Operating Department, Beijing Open University, Chin; Email: xiaomiao517@163.com.

About the Author Jing Gao, Postgraduate Student, Department of Educational Technology, Capital Normal University, China, Email: gaojing11008389@163.com. 


\section{Introduction}

$\mathrm{E}$ -LEARING environment, which carries the whole process of online learning, has been proposed to have a big impact on students' physical health and has aroused widespread concerns. At present, the impact of E-learning environment on students' physical health has been becoming a hot issue in China with the rapid development of information technology. For this reason, the Ministry of Education of China has specially set up a project, and Capital Normal University was in charge of the one-year large-scale research with joint conduction of the General Administration of Sport of China, Beijing Sports University and other research institutes to formulate the "E-learning Environment User Manual" and provide parents, schools and communities with important guidelines for protecting the health of elementary and middle school students.

\section{A Literature Review on the Relationship between E-learning Environment and Students' Physical Health}

National Students' Physical Health Survey Results in 2014 showed that the rate of adolescents with poor eyesight in China was still high and tendened to become younger; and the rate of obesity among students of all ages continued to rise; the physical fitness, such as flexibility and strength, continued to show a steady and positive trend, but the level of physical health was worsening (General Administration of Sport of China, 2015). On the basis of these phenomena, parents and communities start to doubt the use of smart devices and E-learning environment.

The adverse effects of hardware environment of online learning on students' physical health have been confirmed that long-term use of computers and other electronic devices cause the absorption of electromagnetic waves and harmful gases released by these devices produced harmful effect on adolescents' health (Zhang et al., 2018). A web-based trial of dry eye disease showed that about $36.2 \%$ of netizens experienced five or more types of dry eye symptoms, and network using had an ascertained effect on eyes and excessive use would damage eyesight (Wang et al., 2018). Cumulating data have shown that mobile internet addiction could cause cervical spine stiffness, obesity, decreased vision, insufficient thoracic blood and cardiopulmonary function

\footnotetext{
About the Author: Hong Feng, Associate Professor, Department of Educational Technology, Capital Normal University, China; Email: hg_feng_cnu@126.com.

Zhixin Zhang, Lecturer, Department of Educational Technology, Capital Normal University, China; Email: zhangzx517@163.com.
}

Funding: This paper is a phased research result of the Ministry of Education-China Mobile Research Fund (2013) research project "Empirical Study on the Impact and Intervention of Internet Learning Environment on Students' Physical and Mental Health" (Project No. MCM20130602).

Conflict of Interests: None. 
decline in adolescents; and computer internet addiction could cause decreased vision, obesity, insomnia and inappropriate eating behavior in adolescents; and the sub-health status of adolescents was closely related to the time they spent on internet (Wang et al., 2017).

Other peer researches such as the American Academy of Ophthalmology also believed that increased frequency of computer use would lead to eye fatigue, redness, blurred vision, myopia and other various eye symptoms and potential visual impairment that depend on several factors including using time, viewing distance, sitting posture, computer screen (Cai, 2017). Frequently watching computer screens could cause eye irritation, blurred vision, fatigue and headache (Kozeis, 2009). The most common symptoms from the use of computers were eye burning and muscle pain, which are strongly related to the duration of computer use (Taptagaporn, 2009).

Although many recent studies had observed the influence of e-learning environment on students' eyesight, obesity, cardiopulmonary function and so on, most of them were based on subjective judgments and lacked solid scientific evidence, and it is still difficult to explain scientifically how the e-learning environment affects the health of students. Therefore, we intended in this study to answer following two questions:

- Does the e-learning environment have any impact on the physical health of elementary and middle school students?

- In order to prevent e-learning environment from affecting students' health, how long should they use e-learning environment every day?

\section{Research Implementation}

\section{Research Tools}

In this study, eyesight, height and weight, sit and reach of the subjects were tested using standard logarithmic visual acuity scale (GB 11533-1989, Shanghai Aice Electronic Technology Company Limited), electronic height and weight meter (HGM-702, Henan Shengyuan Industrial Company Limited), sit and reach tester (HHTC/ZT100, Beijing Huaxia Huihai Technology Company Limited). Body Mass Index equals weight divided by height squared, and respiratory rate was recorded and calculated with stopwatch (GY12001, Shanghai Stopwatch Factory).

\section{Research Objects}

A total of 783 students from two middle schools and four elementary schools in Beijing city and Shandong province were selected by Convenient Sampling method, taking into the differences in economy, urban and rural locations, and quality of students etc. These students were the second, third, fourth and seventh graders, and all belonged to 21 experimental classes where the e-learning using was more than one semester and one control class where no e-learning environment at all.

\section{Data Collection and Processing}


From September to October 2014, the first test data were obtained; from September to October 2015, the second test data were obtained.

This study investigated the use of e-learning environment, including:

- Time length of using "electronic whiteboard" or "touch integrated machine" in class;

- Time length of using "tablet" or "online notebook" in class;

- Time length of using "tablet" or "online notebook" outside classroom.

According to the preliminary statistics, the duration of using of e-learning environment in the experimental and control classes was between 0 and 30 class hours per week. According to the time length of e-learning environment using each week and excluding missing values, the students in both experimental and control classes were divided into two groups with equal numbers. The first group used less e-learning environment ( 0 - 13 class hours each week) and the second group over-utilized e-learning environment (14 - 30 class hours each week), and the grade distribution of the two groups was the same. In this study, SPSS 19.0 was used for data processing and statistical analysis. Covariance Analysis was used to explore the impact of time length of elearning on the health of elementary and middle school students, as well as the extent to which students of different genders and grades were affected. According to the Mean Values Adjusted by Covariance, it was concluded that how long e-learning environment was used was beneficial to the health of the students through analysis.

\section{Analysis and Research Results}

\section{Eyesight}

\section{The Relationship between Eyesight and the Time Length of e-learning Environment Using Each Week in Elementary and Middle School Students}

In this study, the time length of e-learning environment using each week in the sample students was the independent variable, the second test of the left eyesight was the dependent variable, and the first test of the left eyesight was the covariate. The homogeneity test results of the regression coefficients in the group showed that the interaction between the independent variables and the covariance did not reach a significant level $\left(\mathrm{F}_{(1,479)}=0.358, \mathrm{P}>0.05\right)$, indicating that the covariance in each group was consistent with the linear relationship of the dependent variables and the Covariance Analysis need to be continued. In order to correctly estimate the effect of the interaction between independent variable and covariance, this study removed the interaction term and reanalyzed it. In addition, Levene's Variance Homogeneity Test did not reach significant levels $\left(\mathrm{F}_{(1,481)}=0.016, \mathrm{P}>0.05\right)$, showing that there was no significant difference between the discrete cases of the two groups. The test of covariance effect reached a significant level $\left(\mathrm{F}_{(1,480)}=905.533, \mathrm{P}<.01\right)$, satisfying the conditions of the linear relationship. The test of the effect between groups reached a significant level $\left(\mathrm{F}_{(1,480)}=\right.$ $18.940, \mathrm{P}<0.01)$, indicating that the time length of using of e-learning environment 
each week significantly affected the left eyesight of elementary and middle school students.

In the same way, the time length of e-learning environment using each week in elementary and middle school students as the independent variable, the second test of the right eyesight as the dependent variable, and the first test of the right eyesight as the covariate, used the Covariance Analysis method for analysis. The results showed that the time length of E-learning environment using each week significantly affected the right eyesight of elementary and middle school students $\left(\mathrm{F}_{(1,482)}=4.130, \mathrm{P}<0.05\right)$. Comparing the Mean Values Adjusted by Covariance, we found that the left and right eyesight of elementary and middle school students in the group 1 was significantly better than that in the group 2.

\section{Comparison of the Degree of Different Genders Students' Eyesight Affected by the Time Length of E-learning Environment Using Each Week}

In order to explore the extent to which the eyesight of students of different genders was affected by the time length of e-learning environment using each week, we used Covariance Analysis to analyze the sample data of male and female students, respectively. The results of the male sample showed that the effect between the left eyesight reached a significant level $\left(\mathrm{F}_{(1,239)}=10.214, \mathrm{P}<0.01\right)$, while the the effect between the right eyesight did not reach a significant level $\left(\mathrm{F}_{(1,242)}=0.974, \mathrm{P}>0.05\right)$. The results of Covariance Analysis of female sample data showed that the effect between the left eyesight groups reached a significant level $\left.\left(\mathrm{F}_{(1,238}\right)=11.595, \mathrm{P}<0.01\right)$, and the effect between the right eyesight groups also reached a significant level $\left(\mathrm{F}_{(1,237)}=5.933, \mathrm{P}<\right.$ 0.05). In conclusion, the time length of e-learning environment using each week significantly affected the left eyesight of the boys and both left and right eyesight of the girls.

Comprehensive comparison of the Modified Means of Covariance between boys and girls, we found that the left eyesight of boys in the group 1 was significantly better than that of boys in the group 2, while the difference between the two groups of boys in the right eyesight was not significant; both left and right eyesight of girls in the group 1 was significantly better than that of girls in the group 2 .

\section{Comparison of the Degree of Different Semesters Students' Eyesight Affected by the Time Length of E-learning Environment Using Each Week}

In order to explore the extent to which the eyesight of students of different semesters was affected by the time length of e-learning environment using each week, we used Covariance Analysis to analyze the sample data of middle school students and elementary school students, respectively. The results of Covariance Analysis of middle school students data showed that the effect between the left eyesight groups reached a significant level $\left(\mathrm{F}_{(1,108)}=10.529, \mathrm{P}<0.01\right)$, while the effect between the right eyesight groups did not reach a significant level $\left(\mathrm{F}_{(1,105)}=0.779, \mathrm{P}>0.05\right)$. The results of Covariance Analysis of elementary school students data showed that: the effect between the left eyesight groups reached a significant level $\left(\mathrm{F}_{(1,369)}=7.646, \mathrm{P}<0.01\right)$, and the effect between the right eyesight groups also reached a significant level $\left(\mathrm{F}_{(1,374)}=4.488, \mathrm{P}<\right.$ 
0.05). In sum, the time length of e-learning environment using each week significantly affected the left eyesight of the middle school students and both left and right eyesight of the elementary school students.

Comprehensive comparison of the Modified Mean of Covariance between middle school students and elementary school students, we found that the left eyesight of middle school students in the group 1 was significantly better than that of the middle school students in the group 2, while the difference between the two groups of middle school students in the right eyesight was not significant; the left and right eyesight of elementary school students in the group 1 was significantly better than that of the elementary school students in the group 2.

\section{Respiratory Rate}

The Relationship between Respiratory Rate and the Time Length of E-learning Environment Using Each Week in Elementary and Middle School Students

In this study, the time length of e-learning environment using each week was the independent variable, the second test of students' respiratory rate was the dependent variable, and the first test of students' respiratory rate was the covariate. The results showed that the effect between the two groups did not reach a significant level $\left(\mathrm{F}_{(1,512)}=0.734\right.$, $\mathrm{P}>0.05)$, indicating that the time length of e-learning environment using each week had no significant effect on the respiratory rate of elementary and middle school students.

\section{Comparison of the Degree of Different Genders Students' Respiratory Rate Affected by the Time Length of E-learning Environment Using Each Week}

In order to explore the extent to which the respiratory rate of students of different genders was affected by the time length of e-learning environment using each week, we used Covariance Analysis to analyze the data of male and female students, respectively. The results of Covariance Analysis of male sample data showed that the effect between groups did not reach a significant level $\left(\mathrm{F}_{(1,264)}=0.085, \mathrm{P}>0.05\right)$. The results of Covariance Analysis of female data also showed that the effect between groups did not reach a significant level $\left(\mathrm{F}_{(1,245)}=1.157, \mathrm{P}>0.05\right)$. In sum, the time length of e-learning environment using each week had no significant effect on the respiratory rate of boys and girls.

\section{Comparison of the Degree of Different Semesters Students' Respiratory Rate Affected by the Time Length of E-learning Environment Using Each Week}

In order to explore the extent to which the respiratory rate of students of different semesters was affected by the time length of e-learning environment using each week, we used Covariance Analysis to analyze the data of middle school students and elementary school students, respectively. The results of Covariance Analysis of middle school students data showed that the effect between groups did not reach a significant level $\left(\mathrm{F}_{(1,112)}\right.$ $=0.138, \mathrm{P}>0.05)$. The results of Covariance Analysis of elementary school students 
data also showed that the effect between groups did not reach a significant level $\left(\mathrm{F}_{(1,397)}\right.$ $=0.459, \mathrm{P}>0.05)$. In sum, the time length of e-learning environment using each week had no significant effect on the respiratory rate of middle school and elementary school students.

\section{Body Mass Index}

The Relationship between Body Mass Index and the Time Length of E-learning Environment Using Each Week in Elementary and Middle School Students

In this study, the time length of e-learning environment using each week in the students was the independent variable, the second test of the students' Body Mass Index was the dependent variable, and the first test of the students' Body Mass Index was the covariate. The results showed that the effect between groups did not reach a significant level $\left(\mathrm{F}_{(1,501)}=0.462, \mathrm{P}>0.05\right)$, indicating that the time length of using E-learning environment each week had no significant effect on the Body Mass Index of elementary and middle school students.

\section{Comparison of the Degree of Different Genders Students' Body Mass Index Affected by the Time Length of E-learning Environment Using Each Week}

In order to explore the extent to which the Body Mass Index of students of different genders was affected by the time length of e-learning environment using each week, we used Covariance Analysis to analyze the data of male and female students, respectively. The results of Covariance Analysis of male data showed that the effect between groups did not reach a significant level $\left(\mathrm{F}_{(1,252)}=0.039, \mathrm{P}>0.05\right)$. The results of Covariance Analysis of female data also showed that the effect between groups did not reach a significant level $\left(\mathrm{F}_{(1,246)}=1.722, \mathrm{P}>0.05\right)$. In sum, the time length of using of the Elearning environment each week had no significant effect on the Body Mass Index of both boys and girls.

\section{Comparison of the Degree of Different Semesters Students' Body Mass Index Affect- ed by the Time Length of E-learning Environment Using Each Week}

In order to explore the extent to which the Body Mass Index of students of different semesters was affected by the time length of e-learning environment using each week, we used Covariance Analysis to analyze the data of middle school students and elementary school students, respectively. The results of Covariance Analysis of middle school students data showed that the effect between groups reached a significant level $\left(\mathrm{F}_{(1,111)}\right.$ $=5.218, \mathrm{P}<0.05)$. But the results of Covariance Analysis of elementary school students data showed that the effect between groups did not reach a significant level $\left(\mathrm{F}_{(1,387)}=\right.$ 0.095, $\mathrm{P}>0.05)$. In sum, the time length of e-learning environment using each week significantly affected the Body Mass Index of middle school students, but had no significant effect on Body Mass Index of elementary school students.

\section{Sit and Reach}




\section{The Relationship between Sit and Reach and the Time Length of E-learning Envi- ronment Using Each Week in Elementary and Middle School Students}

In this study, the time length of e-learning environment using each week in the students was the independent variable, the second test of the students' sit and reach was the dependent variable, and the first test of the students' sit and reach was the covariate. The results showed that the effect between the two groups did not reach a significant level $\left(F_{(1,513)}=0.032, \mathrm{P}>0.05\right)$, indicating that the time length of e-learning environment using each week had no significant effect on the sit and reach of elementary and middle school students.

\section{Comparison of the Degree of Different Genders Students' Sit and Reach Affected by the Time Length of E-learning Environment Using Each Week}

In order to explore the extent to which the sit and reach of students of different genders was affected by the time length of e-learning environment using each week, we used Covariance Analysis to analyze the data of male and female students, respectively. The results of Covariance Analysis of male data showed that the effect between the two groups did not reach a significant level $\left(\mathrm{F}_{(1,263)}=0.844, \mathrm{P}>0.05\right)$. The results of Covariance Analysis of female data also showed no significant difference $\left(\mathrm{F}_{(1,247)}=3.352, \mathrm{P}>\right.$ 0.05). In sum, the time length of e-learning environment using each week had no significant effect on the sit and reach of boys and girls.

\section{Comparison of the Degree of Different Semesters Students' Sit and Reach Affected by the Time Length of E-learning Environment Using Each Week}

In order to explore the extent to which the sit and reach of students of different semesters was affected by the time length of e-learning environment using each week, we used Covariance Analysis to analyze the data of middle school students and elementary school students, respectively. The results of Covariance Analysis of middle school students data showed that the effect between the two groups did not reach a significant level $\left(\mathrm{F}_{(1,112)}=0.001, \mathrm{P}>0.05\right)$. The results of Covariance Analysis of elementary school students data also showed no significant difference $\left(F_{(1,398)}=1.193, P>0.05\right)$. In sum, the time length of the E-learning environment using each week had no significant effect on the sit and reach of both middle school and elementary school students.

\section{Conclusion and Suggestion}

From the results of Covariance Analysis above, our study demonstrated following conclusions. First, according to the full sample analysis, the time length of e-learning environment using each week by elementary and middle school students had a significant impact on their eyesight, but not on respiratory rate, body mass index, and sit and reach. However, we cannot rule out other confounding factors that were difficult to be controlled. In terms of eyesight, students with less use of the e-learning environment showed better eyesight than that of the over-utilized ones. After further refinement of the e-learning environment using to a daily basis, we found that the time length of daily 
using of E-learning environment for elementary and middle school students should be controlled less than 1.5 hours.

Second, after comparing the effect of the time length of e-learning environment using each week with different genders, we received consistent results with the whole sample analysis in terms of respiratory rate, body mass index, and sit and reach. To the eyesight, the time length of e-learning environment using each week significantly affected both left and right eyesight of girls, but only on the left eyesight of the boys, indicating that girl's eyesight was more sensitive and was more easily affected than that of boy's even under the same condition. Clinical studies have found that $53 \%-85 \%$ of people had right eye as their dominant eye (Miao et al., 2007). In the binocular eyesight activity, the dominant eye occupies more space and has better clarity and development than the auxiliary eye (Zang, 2012). The left eye might be more vulnerable to damage as an auxiliary eye in e-learning environment - of course, this proposed statement is yet, confirmed by further research.

Third, according to the comparison results of different semesters affected by the time length of e-learning environment using each week, we got consistent results with the whole sample analysis in terms of respiratory rate, body mass index, and sit and reach. Regarding the eyesight, the time length of e-learning environment using each week significantly affected both left and right eyesight of elementary school students, but only had a significant impact on the left eyesight of the middle school students indicating that students from elementary school were more sensitive in their eyesight and more easily to be affected than those from middle school. In addition, the time length of e-learning environment using each week significantly affected the body mass index of middle school students, but had no significant effect on that of the elementary school students. This may be because middle school students were at a critical stage of growth and development, so their body mass indexes were more susceptible to the time length of e-learning environment using.

Based on these findings, we proposed that:

- Primary and middle schools should arrange reasonable number of sessions of the e-learning environment using, and increase the extracurricular sports activities;

- Teachers should reasonably use a variety of media in teaching, but could not rely only on modern electronic media such as electronic whiteboards and internet notebooks;

- Parents should take more time to accompany their children and strictly control their extracurricular use of electronic devices and set themselves as role models. All in all, schools, teachers, and parents need to cooperate to create a good elearning environment for students and control the time length of the daily use of e-learning environment to less than 1.5 hours. 


\section{References}

Cai, P. (2017) Research on the influence of Network Learning Environment on the eyesight of elementary and middle school Students. Wuhan, P.R.China: Central China Normal University, 4.

General Administration of Sport of China. (2015) Results of the 2014 national student physical health survey. Chinese Journal of School Health; 12:4.

Kozeis, N. (2009) Impact of computer use on children's vision. Hippokratia; (4): 230231.

Miao, A.Z., Zhou, X.T. (2007) Advances in the study of dominant eye. Chinese Journal of Ocular and Otorhinolaryngology; (1): 61- 62 .

Taptagaporn, S. (2009) Computer use among children: Health and social impacts. Proceedings of 17th World Congress on Er- gonomics. Beijing, P.R.China: International Ergonomics Association, 6.

Wang, W., Lei, L., Yang, J.H. (2018) The influence of Internet on the physical and mental health of netizens. Journal of Shanxi Datong University (Natural Science Edition); (2): 69-73, 79.

Wang, Z.S., Wei, Y. (2017) The influence of Internet addiction on adolescent health. Youth Sports; (1): 120-121, 53.

Zang, Y.J. (2012) Study on characteristic parameters affecting the comfort of stereo image. Tianjin, P.R. China: Tianjin University, 5- 6 .

Zhang, Z.X., Gao, J., Feng, H., Xie, J.R., Zhang, X.Y. (2018) Review on the impact of online learning on adolescents' physical and mental health. Modern Distance Education; (3): 59- 65.

Received: 23 July 2019

Revised: 14 August 2019

Accepted: 05 September 2019

The Chinese version of this article has been published in Modern Edu Technol, 29(3):60-65. The English version has been authorized for being publication in BECE by the author(s) and the Chinese journal.

高景, 刘静, 冯红, 张志新. 网络学习环境使用时长与中小学生身体健康的关系探究. 现代教 育技术, 2019; 29(3):60-65. 